\title{
Experimental study on the effect of aerobic exercise on body fat reduction in patients with type 2 diabetes.
}

\author{
Xiu-Chang Zhang, Chul-Hyun Kim* \\ Soonchunhyang University, Asan, Chungnam, Korea
}

\begin{abstract}
Objective: To study the clinical effects of aerobic exercise on patients with type 2 diabetes.

Methods: 74 cases of patients with type 2 diabetes were selected and divided into two groups based on different intervention methods.

Results: Before the intervention, the differences of body fat, blood fat and blood glucose changes between both groups were not statistically significant, with $P>0.05$.After the intervention, the body fat of the experimental group was significantly reduced. The change difference was significantly better than that of the control group. At the same time, the changes of blood fat and blood glucose of the experimental group were significantly better than the control group, with $\mathbf{P}>0.05$.

Conclusion: Aerobic exercise intervention can improve the blood fat and blood glucose indicators of patients with type 2 diabetes and reduce their body fat and weight, which worth clinical promotion with significant research value.
\end{abstract}

Keywords: Aerobic exercise, Type 2 diabetes, Body fat, Blood glucose, Blood fat.

Accepted on May 04, 2017

\section{Introduction}

Type 2 diabetes is a clinically common diabetes, which is often seen in the middle-aged and elderly people. People's lifestyle and eating habits change along with their age. More highcalorie and high-fat food and less exercise makes it easy to be fat and thus leads to type 2 diabetes. In clinical treatment, injectable insulin and drugs that stimulate the production of insulin, including metformin, rosiglitazone, acarbose, and gliclazide are often used to control diabetes. However, besides poor effect on blood glucose control, the utilization of insulin drugs may lead to some adverse reactions. In this case, by strengthening physical exercise to reduce body fat, patients with type 2 diabetes will be independent from insulin drugs, with better treatment effect. In view of this, we adopted aerobic exercise intervetnion to patients with type 2 diabetes and achieved significant research results.

\section{Materials and Methods}

\section{General information}

A total of 74 cases of patients with type 2 diabetes from May 2014 to May 2016 were selected. Patients in both groups met the clinical diagnostic criteria of type 2 diabetes [1]. All patients were randomly divided into control group and experimental group. There were 37 cases in the control group, 21 were male and 16 were female. The age range was 32 to 54 years old and the average age was $(42.09 \pm 0.91)$. There were 37 cases in the experimental group, 25 were male and 15 were female. The age range was 37 to 68 years old and the average age was $(51.46 \pm 1.04)$. After the comparison of the general information, there was no significant difference between both groups, with $\mathrm{P}>0.05$.

\section{Intervention Methods}

Patients in the control group were given routine treatment and nursing. Patients in the experimental group were given aerobic exercise intervention. Health care workers learnt the actual condition and interests of patients with type 2 diabetes through close communication to develop targeted aerobic exercise plans based on their age, health condition, and interest [2]. Aerobic exercise like Taiji and square dance can be adopted by elder patients. In this way, we could stimulate their interest in exercise to improve their amount of exercise and reduce body fat. Aerobics and jogging could be adopted by younger patients. At the same time, health care workers guided the exercise of patients based on step-by-step principle. The heart rate was controlled at about $60 \%$. Single aerobic exercise lasted about 60 minutes for 3 times a week [3].

\section{Indicator Observation}

After the intervention, changes in body fat, blood glucose, and blood fat of both groups were compared.

Body fat: Abdominal skinfold thickness, body fat rate, and visceral fat area before and after the intervention were measured [4]. 
Blood glucose: Fasting blood glucose and two hours after meal blood glucose before and after the intervention were measured [5].

Blood lipid: Total cholesterol, high and low density cholesterol, and triglycerides before and after the intervention were measured [6].

\section{Data analysis}

SPSS 13.0 was used for statistical data analysis. The change of body fat, blood glucose, and blood lipid of both groups were taken as measurement data and expressed by mean value \pm standard deviation. $\mathrm{T}$ test was adopted. $\mathrm{P}<0.05$ means the difference was statistically significant.

\section{Results}

\section{Comparison of body fat change of both groups before and after the intervention}

Based on the statistical analysis, before the intervention, the body fat change of both groups showed no significant difference, with $\mathrm{P}>0.05$; After the intervention, the body fat of the experimental group reduced significantly. The change was significantly better than that of the control group. The difference was statistically significant, with $\mathrm{P}<0.05$ (Table 1).

Table 1. Comparison of body fat change of both groups before and after the intervention $(x \pm s)$.

\begin{tabular}{|c|c|c|c|c|c|}
\hline Group & $\mathbf{n}$ & Time & Abdominal Skinfold Thickness & Body fat rate & Visceral Fat Area \\
\hline \multirow{2}{*}{ Experimental group } & \multirow{2}{*}{37} & Before Intervention & $29.75 \pm 7.94$ & $31.34 \pm 8.23$ & $114.52 \pm 29.76$ \\
\hline & & After Intervention & $24.33 \pm 5.47$ & $23.91 \pm 9.08$ & $95.31 \pm 28.49$ \\
\hline \multirow{2}{*}{ Control group } & \multirow{2}{*}{37} & Before Intervention & $28.47 \pm 7.06$ & $31.77 \pm 8.54$ & $114.99 \pm 31.57$ \\
\hline & & After Intervention & $27.87 \pm 4.01$ & $32.06 \pm 7.12$ & $115.38 \pm 336.33$ \\
\hline
\end{tabular}

Note: Compared with the control group, $\mathrm{P}<0.05$.

\section{Blood glucose change of both groups before and after the intervention}

Before the intervention, the blood glucose change of both groups showed no significant difference, with $\mathrm{P}>0.05$; After the intervention, the blood glucose of the experimental group reduced significantly. The change was significantly better than that of the control group. The difference was statistically significant, with $\mathrm{P}<0.05$ (Table 2).

\section{Blood lipid change of both groups before and after the intervention}

Based on the statistical analysis, before the intervention, the blood lipid change of both groups showed no significant difference, with $\mathrm{P}>0.05$; After the intervention, the blood lipid of the experimental group reduced significantly. The change was significantly better than that of the control group. The difference was statistically significant, with $\mathrm{P}<0.05$ (Table 3 ).

Table 2. Change of blood glucose of both groupsbefore and after the intervention $(x \pm s)$.

\begin{tabular}{|c|c|c|c|c|c|}
\hline Group & $\mathbf{n}$ & Time & FBG & $\begin{array}{l}\text { Postprandial } \\
\text { Glucose }\end{array}$ & Blood \\
\hline \multirow{2}{*}{$\begin{array}{l}\text { Experiment } \\
\text { al group }\end{array}$} & \multirow{2}{*}{$\begin{array}{l}3 \\
7\end{array}$} & $\begin{array}{l}\text { Before } \\
\text { Intervention }\end{array}$ & $8.03 \pm 1.04$ & $12.33 \pm 1.97$ & \\
\hline & & After Intervention & $4.79 \pm 2.14$ & $5.29 \pm 2.77$ & \\
\hline \multirow{2}{*}{$\begin{array}{l}\text { Control } \\
\text { group }\end{array}$} & \multirow{2}{*}{$\begin{array}{l}3 \\
7\end{array}$} & $\begin{array}{l}\text { Before } \\
\text { Intervention }\end{array}$ & $7.79 \pm 1.58$ & $13.08 \pm 2.15$ & \\
\hline & & After Intervention & $7.03 \pm 1.46$ & $7.06 \pm 2.38$ & \\
\hline
\end{tabular}

Note: Compared with the control group, $\mathrm{P}<0.05$.

Table 3. Change of blood lipid of both groupsbefore and after the intervention $(x \pm s)$.

\begin{tabular}{|c|c|c|c|c|c|c|}
\hline Group & $\mathbf{n}$ & Time & Low Density Cholesterol & High Density Cholesterol & Triglycerides & Total Cholesterol \\
\hline \multirow{2}{*}{ Experimental group } & \multirow{2}{*}{37} & Before Intervention & $4.01 \pm 1.30$ & $2.13 \pm 1.09$ & $2.64 \pm 1.23$ & $5.69 \pm 1.34$ \\
\hline & & After Intervention & $3.47 \pm 0.22$ & $1.26 \pm 1.68$ & $1.43 \pm 0.16$ & $4.23 \pm 1.12$ \\
\hline \multirow{2}{*}{ Control group } & \multirow{2}{*}{37} & Before Intervention & $4.36 \pm 1.05$ & $2.16 \pm 1.20$ & $2.54 \pm 0.97$ & $5.79 \pm 1.97$ \\
\hline & & After Intervention & $4.25 \pm 1.02$ & $2.21 \pm 1.53$ & $2.31 \pm 0.25$ & $5.41 \pm 1.09$ \\
\hline
\end{tabular}

Note: Compared with the control group, $\mathrm{P}<0.05$. 


\section{Conclusion}

The abdominal fat accumulation of patients with type 2 diabetes leads to severe complications which threatens patients safety and health. It has been recognized by health care experts that targeted aerobic exercise plan can improve patients' exercise initiative and their obesity symptoms, reduce body fat, and help improve various indicators including blood lipid and blood glucose, to improve the treatment effect [7].

In this experimental study, 74 cases of patients with type 2 diabetes were randomly divided into control group and experimental group. 37 cases of patients in the control group were given routine treatment and nursing. 37 cases of patients in the experimental group were given aerobic exercise intervention. After the intervention, the body fat of the experimental group was significantly reduced. The change was significantly better than that of the control group. At the same time, the clinical change of blood lipid and blood glucose of the experimental group were significantly better than the control group. The differences were statistically significant, with $\mathrm{P}>0.05$.

In summary, aerobic exercise intervention can improve the blood fat and blood glucose indicators of patients with type 2 diabetes and reduce their body fat and weight and improve their treatment effect and life quality, which worth clinical promotion with significant research value.

\section{References}

1. Xiao X, Yunfa L. Effect of different exercises on patients with type 2 diabetes. China School Physical Education (High Education) 2016; 3: 89-93.
2. Yifei G, Xuefeng L, Min L. The influence to glucose and lipid metabolism and insulin resistance and leptin of postprandial aerobic exercise in newly diagnosed obese type 2 diabetes. Chinese J Difficult Complicated Cases 2016; 15: 706-709.

3. Chunxiao L. The impact on quality of life by prescriptive exercise and diet intervention in patients with diabetes mellitus. China Modern Med 2015; 22: 40-41.

4. Jiaming F, Zhimin L, Daoli D. Effect of rejuvenation medical exercise on the serum levels of blood glucose and blood lipid of elderly patients type 2 diabetes. Progress Modern Biomed 2015; 15: 5291-5294.

5. Yuhui L. Influence of Aerobic Exercise on Blood Lipid of Patients with Type 2 Diabetes. World Latest Med Info 2015; 15: 46-47.

6. Xiaoxi S. Effect of low intensity aerobic exercise on blood glucose in type 2 diabetes. Chinese J Gerontol 2015; 35: 4595-4597.

7. Suying L. Effect of exercise on blood glucose of patients with type 2 diabetes. Jilin Med J 2014; 35: 3834-3835.

\section{*Correspondence to}

Chul-Hyun Kim

Soonchunhyang University

Korea 